\title{
Obey or Play: Asymptotic Equivalence of Slotted Aloha with a Game Theoretic Contention Model
}

\author{
Jaume Barcelo, Hazer Inaltekin, Member, IEEE, and Boris Bellalta
}

\begin{abstract}
Contention control is an important means to mitigate adverse affects of multiple access interference on transmitted data packets in wireless communication networks. Most practical contention control mechanisms, e.g., IEEE 802.11 and slotted Aloha, rely on the assumption that all users abide with the rules of the protocol. There is also a growing body of work employing game theoretic techniques to gain new design insights for controlling contention when users act selfishly to maximize their perceived performance, as well as to reverse/forward engineer existing contention control protocols. In this paper, it is shown that the asymptotic behaviour of the slotted Aloha protocol when the number of contenders grows large coincides with a Nash equilibrium that is derived from a game theoretic contention model. Furthermore, the relationship between the design parameters in both approaches is established. In particular, it is shown that the back-off exponent in the slotted Aloha based contention control is linearly related to the cost of a collision in the game theoretic approach.
\end{abstract}

Index Terms-Slotted Aloha, game theory, contention control, medium access control.

\section{INTRODUCTION}

A key performance limiting criterion in wireless communication networks is the level of multiple access interference (MAI). This is mainly due to the broadcast nature of wireless channels shared by multiple mobile stations simultaneously. Therefore, a typical mobile station in a wireless network often ends up hearing data packets not intended for itself, which, in turn, causes interference for the useful data traffic and reduces data rates at this station. This paper focuses on layer 2 contention control protocols to manage MAI in wireless networks, and provides new insights into contention control by establishing connections between a slotted Aloha based contention control protocol and a game theoretic contention control model.

Proper management and mitigation of MAI in wireless networks is a long-standing design challenge [1]. To this end, various contention control protocols including IEEE 802.11, CSMA/CA and slotted Aloha were proposed as effective means to share wireless medium among multiple mobile stations. The traditional way to analyze the resulting network

J. Barcelo is with the Dept. de Ingenieria Telematica, Universidad Carlos III de Madrid, Spain (email:jaume.barcelo@uc3m.es).

H. Inaltekin is with the ARC Special Research Centre for Ultra-Broadband Information Networks, Dept. of Electrical and Electronic Engineering, University of Melbourne, Australia (email:hazeri@unimelb.edu.au).

B. Bellalta is with the Dept. de Tecnologies de la Informacio i les Comunicacions, Universitat Pompeu Fabra, Spain (email:boris.bellalta@upf.edu). (C) 2011 IEEE. Personal use of this material is permitted. Permission from IEEE must be obtained for all other uses, in any current or future media, including reprinting/republishing this material for advertising or promotional purposes, creating new collective works, for resale or redistribution to servers or lists, or reuse of any copyrighted component of this work in other works. performance under a contention control protocol is to assume that participating stations strictly obey to a simple set of rules that guarantee fair and efficient access to the shared wireless channel. However, with the advent of increasingly autonomous and heterogeneous wireless devices, it is possible to envisage scenarios in which some devices misbehave and obtain an unfair share of the channel resources [2]. In these circumstances, a possible solution involves probabilistic techniques to detect misbehaving communication nodes as in [3], and then punish them to prevent malicious activity from these nodes in the future. However, this approach may be prohibitive if there is a large number of nodes in the network.

An alternative approach is to design the contention control mechanism from a game theoretic point of view, e.g., see [4], [5] and [6]. Generally speaking, game theory [7] studies strategic situations in which rational and intelligent players take selfish decisions in order to maximize their utilities. In the context of network design and analysis, game theory and the related field of mechanism design have the potential to guide engineering efforts in designing efficient network protocols by providing a bottom-up analytical and principled approach, and to verify resulting collective network behavior through equilibrium analyses. In particular, if contention protocols operate at Nash equilibria, none of the participating players (the contending stations) has an incentive to unilaterally deviate from the protocol. Furthermore, it is shown in [4] that game theoretic approach also provides new insights to reverse/forward engineer existing contention control protocols by establishing connections between the design parameters of the game theoretic model and those of contention control protocols under consideration. In addition to new insights into contention control design robust against misbehavior, this paper also contributes to reverse/forward engineering efforts of layer 2 contention control protocols by revealing such a connection between an exponential back-off based slotted Aloha protocol and the game theoretic contention control protocol in [5].

In this paper, we focus on collision channels [8], and assume that collisions occur when more than one stations try to access the channel simultaneously. ${ }^{1}$ The packets that collide are retransmitted later, and the stations who participated in the collision suffer an additional penalty. In the slotted Aloha protocol considered in this paper, the transmission probability of a colliding station is divided by $r>1$ until the next successful transmission. On the other hand, the stations involved in the collision must pay a cost $c>0$, which determines

\footnotetext{
${ }^{1}$ Our analysis in this paper is expected to generalize to wireless channels with multipacket reception [9], but we do not consider such extensions here.
} 
channel access probabilities in the game theoretic contention control protocol. This cost is relative to the benefit obtained from a successful transmission, which is normalized to 1 and, for example, it can be interpreted as the energy cost of a transmission in an energy-limited scenario.

$r$ and $c$ parameters in both approaches are expected to be related to each other. After a collision in the slotted Aloha protocol, the average number of slots that will elapse before a successful transmission attempt is multiplied by $r \in \mathbb{N}$. On the other hand, a station suffers from $c \in \mathbb{N}$ units of utility reduction after a collision in the game theoretic approach, which implies $c+1$ successful transmission attempts to reap a net benefit of one. This means that the average number of slots that will elapse before the station obtains a net benefit of one is increased by a factor of $c+1$. Intuitively, the penalty suffered by a station by dividing its transmitting rate by $r$ in the slotted Aloha protocol should be equivalent to $c+1$ units of utility to be obtained from successful transmissions to wipe out the collision cost in the game theoretic protocol. Here, we make this intuition rigorous by showing that the network throughput obtained in both approaches is the same when $r=c+1$ for large numbers of stations contending for channel access.

The rest of the paper is organized as follows. In Section II, we derive the throughput for the exponential back-off based slotted Aloha protocol when the number of stations contending for channel access grows large. In Section III, we revisit a game theoretic contention control model, and derive its throughput for large station populations. These two expressions explicitly establish the relationship between $r$ and $c$ parameters in both approaches. Finally, Section IV concludes the paper.

\section{Throughput OF THE EXPONENTIAL BACK-OFF Based Slotted Aloha Protocol}

In the exponential back-off based slotted Aloha protocol studied in this paper, we consider $N$ mobile stations that decide to transmit or not in each time slot. They use a transmission probability equal to $\left(r_{0}\right)^{-1}$ for a packet's first transmission attempt, and $\left(r_{0} r^{a}\right)^{-1}$ for a packet's ath retransmission attempt. The parameter $r$ is a design degree of freedom of this slotted Aloha protocol, and it characterizes the behavior of the system when the number of stations is asymptotically large.

To derive the throughput of the Aloha network under consideration, we start by computing the collision probability as

$$
p_{c}=1-(1-\tau)^{N-1},
$$

where $\tau$ is the average transmission probability. The overall system transmission rate can be defined as $G=N \tau$, and the system throughput can be expressed as

$$
S=G\left(1-p_{c}\right)=G\left(1-\frac{G}{N}\right)^{N-1} .
$$

From the definition of the contention control protocol, we can further obtain the expected packet service time as

$$
E[X]=r_{0}+r_{0} p_{c} r+r_{0} p_{c}^{2} r^{2}+\ldots=\frac{r_{0}}{1-p_{c} r} .
$$

We isolate $p_{c}$ to obtain

$$
p_{c}=\frac{1}{r}\left(1-\frac{r_{0}}{E[X]}\right) .
$$

And then, we use the fact that the service time for a given station has to be equal to $N$ over service rate:

$$
E[X]=\frac{N}{S} .
$$

After substituting (5) in (4), we obtain

$$
p_{c}=\frac{1}{r}\left(1-\frac{r_{0} S}{N}\right) \text {. }
$$

Since we are interested in the asymptotic system behavior, we let $N \rightarrow \infty$, and rewrite (6) in the limit as

$$
p_{c}=\frac{1}{r} .
$$

Finally, we obtain the asymptotic global attempt rate, and the system throughput as

$$
G=\ln \left(\frac{r}{r-1}\right)
$$

and

$$
S=\left(\frac{r-1}{r}\right) \ln \left(\frac{r}{r-1}\right),
$$

respectively. These expressions have already appeared in [10] and [11].

\section{Throughrut of the Game Theoretic Contention Control Model}

In the non-cooperative game theoretic contention control model studied in this paper (see also [5] for further details), we consider $N$ mobile stations who decide to transmit or not in each time slot. A station that transmits a packet successfully obtains a utility of 1 [unit], and a station that involves in a collision pays a cost equal to $c$ [units], where $c>0$. If a station chooses not to transmit in a given slot, it obtains a payoff equal to 0 [units]. Users greedily and independently choose their transmission probabilities to maximize their expected utilities. This random access game is known to have many Nash equilibria [5], and we are interested in the unique fully mixed Nash equilibrium (FMNE) at which all stations access the channel with the same transmission probability. Assuming all stations are identical, FMNE corresponds to the fair allocation of communication resources.

At the FMNE, all stations randomize between their transmit and back-off actions, which is possible only if the expected reward for both actions is the same. The observation that the expected payoff of transmitting has to be zero for a tagged station leads to

$$
-c+(1+c)(1-\tau)^{N-1}=0 .
$$

We isolate $\tau$ to obtain the equilibrium transmission probability at the FMNE, which is

$$
\tau=1-\sqrt[N-1]{\frac{c}{1+c}} .
$$


If all stations set their transmission probabilities to $\tau$ given in (11), then (10) is satisfied and none of them has an incentive to deviate.

At the FMNE, the collision probability is given by

$$
p_{c}=1-(1-\tau)^{N-1}=\frac{1}{1+c} .
$$

And the global attempt rate $(G=N \tau)$ in the limit $N \rightarrow \infty$ is

$$
G=\ln \left(\frac{c+1}{c}\right) .
$$

Finally, the throughput offered by the game theoretic model is

$$
S=\frac{c}{1+c} \ln \left(\frac{c+1}{c}\right) .
$$

By inspecting (7)-(9) in Sec. II and (12)-(14) in Sec. III, it can be seen that the global attempt rate (and thus the collision probability and the throughput) of the slotted Aloha based contention control protocol is equivalent to the global attempt rate of the game theoretic contention control model when $r=c+1$ for large numbers of stations contending for channel access.

This behavior is illustrated in Fig. 1 where the aggregate throughputs for slotted Aloha and game theoretic contention control are plotted for different values of $c$ and $r$. A value $r_{0}=10$ has been used in the computation of the throughput of the Aloha network. Note that the influence of this parameter on the system throughput fades away as $N$ grows, and the asymptotic throughput is independent of $r_{0}$.

It can be concluded that the operation of the Aloha protocol when the number of contenders is asymptotically large coincides with one of the Nash equilibria of the game theoretic control if the parameter setting $r=c+1$ is satisfied. In this case, a station has no incentive to unilaterally deviate from the protocol.

From a mechanism design perspective, stations in a slotted Aloha network can be considered as participating in a selfish utility maximization problem described in this paper, and setting the cost parameter in the game theoretic model is equivalent to setting the exponential back-off parameter in the Aloha model. Moreover, even if all stations are assumed to be selfish, there is no loss from the throughput optimality if the utility functions of stations can be forward engineered to drive the system to the desired operating point. We refer interested readers to [9] for a detailed discussion on how to manipulate cost parameters of stations, and how to design utility functions to achieve desired operating points.

\section{Conclusions}

In this paper, we have analyzed a layer 2 contention control protocol for wireless networks both from a traditional design perspective and from the perspective of a game theory based design. In particular, we have focused on an exponential back-off based slotted Aloha protocol and a game theoretic contention control model. We have showed that design parameters in both approaches are intimately related to each other. Specifically, both design approaches achieve the same

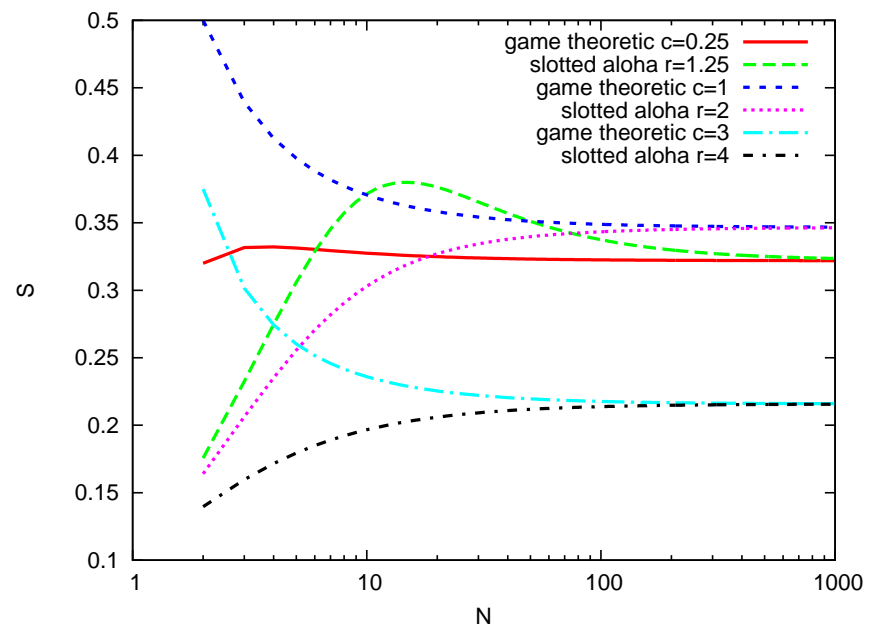

Fig. 1. Comparison of the aggregate throughputs attained by slotted Aloha and game theoretic contention control. $r_{0}=10$.

performance by setting the exponential back-off parameter $r$ in the slotted Aloha protocol to $c+1$, where $c$ is the cost of collision parameter in the game theoretic contention control protocol. This implies that as long as the parameters are set correctly, the Aloha protocol operates at a gametheoretic equilibrium point where no station has an incentive to deviate. Finally, the connections established between both design approaches are also expected to have further implications for reverse/forward engineering efforts of existing layer 2 contention control protocols in wireless networks.

\section{ACKNOWLEDGMENT}

The authors want to express their gratitude to the anonymous reviewers and the editor for their helpful comments.

\section{REFERENCES}

[1] D. Bertsekas and R. Gallager, Data Networks. Upper Saddle River, NJ: Prentice-Hall, 1992.

[2] G. Bianchi, A. Di Stefano, C. Giaconia, L. Scalia, G. Terrazzino, and I. Tinnirello, "Experimental assessment of the backoff behavior of commercial IEEE 802.11 b network cards," in Proc. IEEE INFOCOM, Anchorage, Alaska, May 2007, pp. 1181-1189.

[3] A. Lopez-Toledo and X. Wang, "Robust detection of selfish misbehavior in wireless networks," IEEE J. Sel. Areas Commun., vol. 25, no. 6, pp. 1124-1134, 2007.

[4] J.-W. Lee, A. Tang, J. Huang, M. Chiang, and A. R. Calderbank, "Reverse-engineering MAC: a non-cooperative game model," IEEE J. Sel. Area Commun., vol. 25, no. 6, pp. 1135-1147, Aug. 2007.

[5] H. Inaltekin and S. Wicker, "The analysis of Nash equilibria of the oneshot random-access game for wireless networks and the behavior of selfish nodes," IEEE/ACM Trans. Netw., vol. 16, no. 5, pp. 1094-1107, 2008.

[6] L. Chen, S. H. Low, and J. C. Doyle, "Random access game and medium access control design," IEEE/ACM Trans. Netw., vol. 18, no. 4, pp. 13031316, Aug. 2010.

[7] D. Fudenberg and J. Tirole, Game Theory. Cambridge, MA: MIT Press, 1991.

[8] J. N. Tsitsiklis, "Analysis of a multiaccess control scheme," IEEE Trans. Autom. Control, vol. AC-32, no. 11, pp. 1017-1020, Nov. 1987.

[9] H. Inaltekin, M. Chiang, and H. V. Poor, "Selfish random access over wireless channels with multipacket reception," in Proc. Second International ICST Conference on Game Theory for Networks, Shanghai, China, April 2011.

[10] B. Kwak, N. Song, and L. Miller, "Performance analysis of exponential backoff," IEEE/ACM Trans. Netw., vol. 13, no. 2, pp. 343-355, 2005. 
[11] S. Liew, Y. Zhang, and D. Chen, "Bounded-mean-delay throughput and nonstarvation conditions in Aloha network," IEEE/ACM Trans. Netw., vol. 17, no. 5, pp. 1606-1618, 2009. 